\title{
Myokymia and impaired muscular relaxation with continuous motor unit activity
}

\author{
F. D. L U B L I N, P. T S A I R I , L. J. S T RELETZ, R. A. CHA M B E R , \\ W. F. RIKER, A. V A N POZ N A K, A N D S. W. D U CKETT \\ From the Department of Neurology, Thomas Jefferson University, Philadelphia, Pennsylvania, \\ and Departments of Neurology, Pharmacology, and Anesthesiology, Cornell University Medical College, \\ New York, USA
}

SUMMARY We have studied two cases of the syndrome of myokymia and impaired muscular relaxation with continuous motor unit activity. Both patients complained of muscle twitching, weakness, stiffness, and hyperhydrosis during their illness. Myokymia was present over the entire body in both. On repetitive testing of muscle strength each patient showed initial fatigue followed by increasing strength as he continued his efforts. Both patients improved on phenytoin therapy at high blood levels. Nerve conduction velocities were decreased. Electromyograms showed continuous electrical activity at rest which persisted during sleep and spinal anaesthesia but was diminished by curare. Intravital staining with methylene blue in one case demonstrated sprouting and beading of motor nerve terminals with multiple innervation of muscle fibres. The neurophysiological and pathological findings in these two cases indicate an abnormality of peripheral nerve in this disorder.

Over the last 16 years sporadic reports of the clinical syndrome of myokymia and impaired muscular relaxation (neuromyotonia, pseudomyotonia) have defined a picture of generalised muscular stiffness, continuous undulating contractions of broad strips of muscle (myokymia), hyperhydrosis, and often weakness. The characteristic finding on electromyography is continuous motor unit activity at rest. Several reports have suggested an abnormality of peripheral nerve in this disorder (Wallis et al., 1970; Welch et al., 1972; Irani et al., 1977).

We present clinical, neurophysiological, and pathological findings in two patients with this syndrome. In both, the pattern of weakness is unusual, and is characterised by initial fatigue, followed by gradual increase of strength with repetitive efforts. These cases provide additional evidence of an abnormality of peripheral nerve in this disorder.

\footnotetext{
Address for reprint requests: Dr Fred D. Lublin, Department of Neurology, Jefferson Medical College, 1025 Walnut Street, Philadelphia, Pa 19107, USA.

Accepted 22 November 1978
}

\section{Case reports}

CASE 1

Two years before being studied, this 51 year old man noticed twitching of the muscles of his upper extremities. There followed stiffness of his fingers, slurring of speech, tightness of the facial muscles, numbness and tingling of his fingertips, and increased perspiration. Phenytoin, $300 \mathrm{mg}$ per day, was started with immediate improvement in his symptoms. After six months of phenytoin therapy he noticed difficulty arising from his knees. When walking up a flight of steps, half way up his strength diminished, then gradually returned as he neared the top. His family history and past history were unremarkable. There was no history of exposure to toxins. Physical examination was performed after one week without phenytoin. There was generalised hyperhydrosis. He was mildly dysarthric. Myokymia was present over the face, trunk, and extremities. There was no atrophy or percussion myotonia. The hands were held in carpal spasm, and relaxation of grip was prolonged. Strength was diminished in all muscle groups. On testing strength, there was rapid fatigue after brief effort and improvement with 
rest. If testing was prolonged, after five or six repetitions, the initial fatigue was gradually replaced by increasing power reaching almost normal levels. Tendon and skin reflexes were all absent. Appreciation of pain and vibration was minimally decreased distally.

\section{Laboratory studies}

Results of routine studies were normal. His basal metabolic rate was $+41 \%$. Thyroid function tests were normal. The level of serum creatine phosphokinase (CPK) was 23 units (normal, 0-12). Cerebrospinal fluid protein level was 1.5 and $1.3 \mathrm{~g} / 1$.

\section{Electrophysiology}

Nerve conduction velocities, motor and sensory, were slowed (Table 1). Neuromuscular transmission studies revealed no increment or decrement in evoked responses at stimulation rates of $2,20,50$, or 100 per second. Needle electromyography (EMG) at rest demonstrated continuous electrical activity of full and fractionated motor unit potentials firing at a variable rate of 2 to 50 per second (Fig. 1). Occasional couplets and multiplets were seen. There were no myotonic dis-

Table 1 Nerve conduction studies-case 1

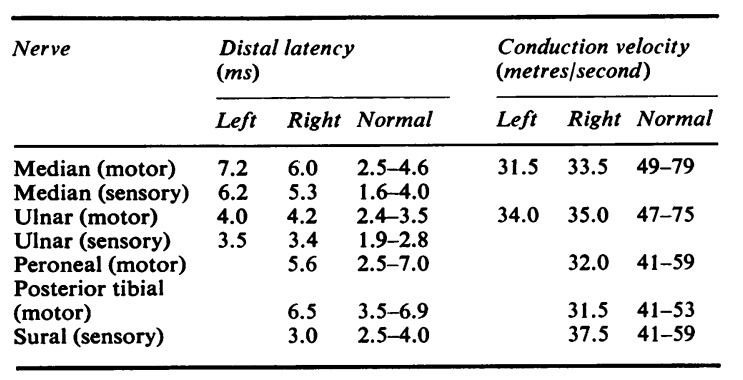

charges on percussion or on movement of the needle. Voluntary muscular contractions produced initially a normal interference pattern. As he tired, there was reduction in the number of motor unit potentials in the interference pattern with each individual unit potential appearing normal. The interference pattern returned to normal as strength improved with exercise. The changes in the interference pattern were not as dramatic as the changes in strength that occurred on repetitive testing. Cessation of voluntary effort was followed by a five to 15 seconds after-discharge, and then by an electrically silent period of one to three minutes before resumption of spontaneous activity. The after-discharge was not seen after cessation of repetitive forceful contractions. Ischaemia decreased the spontaneous activity at rest. The continuous motor unit activity persisted during sleep and spinal anaesthesia. Intravenous curare $(1.8 \mathrm{mg})$ markedly diminished the continual electrical activity. Intravenous edrophonium (Tensilon, $2 \mathrm{mg}$ ) produced a brief burst of electrical activity followed by mild improvement in stiffness for one or two minutes. Larger doses produced no changes.

\section{Histology}

Biopsy samples of external intercostal muscle and nerve were studied with light and electron microscopy and histochemical staining with ATPase, succinyl dehydrogenase, and acid phosphatase. These studies revealed only an abundance of satellite cells in muscle.

\section{Course}

Phenytoin at a dosage of 600 to $700 \mathrm{mg} /$ day with blood levels of 25 to $34 \mu \mathrm{g} / \mathrm{ml}$ resulted in a marked diminution of the myokymia and continuous motor activity, with loss of stiffness. The reflexes and strength returned to normal. The

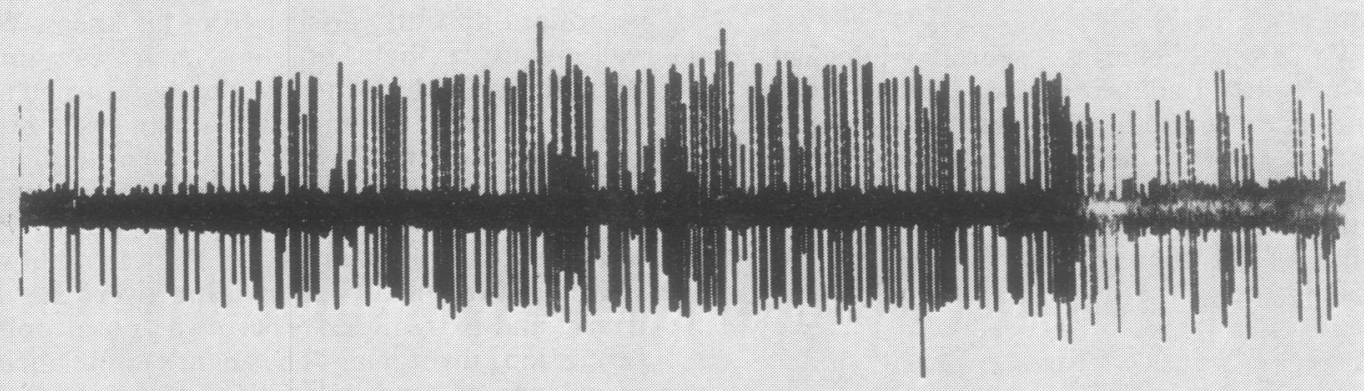

Fig. 1 Needle electromyography at rest in case 1 demonstrating continuous electrical activity of full and fractioned motor unit potentials (amplitude: $100 \mu \mathrm{V} / \mathrm{cm}$; time scale: $100 \mathrm{~ms} / \mathrm{cm}$ ). 
BMR decreased to $-5 \%$. Despite the high blood levels of phenytoin, there were no clinical signs of toxicity. This clinical improvement has lasted for 30 months. Decreasing the dosage of phenytoin results in a return of weakness first and then stiffness.

\section{CASE 2}

A 31 year old man noticed twitching of his facial muscles five years before examination, followed over two years by twitching of the muscles of the trunk and extremities. One year before admission he noticed weakness of his grip and stiffness in his hands and feet, and then progressive generalised weakness. He had noticed some increase in perspiration. He was treated for syphilis 12 years before admissicn, and had been an abuser of intravenous amphetamine. On examination there was moderate hyperinydrosis. He was mildly dysarthric. There was my'kkymia over the face, trunk, and extremities while awake and asleep. There was no percussion myotonia. Strength was markedly diminished in all groups, and he needed help to walk. On testing strength, there was an initial rapid fatigue which improved with rest. When testing was prolonged, strength improved after 10 to 15 repetitions but he never regained full power. Deep tendon reflexes were depressed. Sensory testing was normal.

\section{Laboratory studies}

Routine studies were normal as was his thyroid function. The serum CPK level was 615 units (normal, 0-90). Spinal fluid studies were normal.

\section{Electrophysiology}

Table 2 gives an outline of the nerve conduction velocities and latencies. Motor nerve conduction velocities were slowed significantly. Distal sensory latencies of the right median nerve were normal. Segmental testing of motor conduction velocity of the right ulnar nerve demonstrated slowing throughout its course. Repetitive stimulation of the ulnar nerve produced a decrementing response of $50 \%$ at a stimulus rate of 50 per second (Fig. 2). No change was observed at lower stimulus rates. Needle electromyography revealed continuous motor unit activity at rest (Fig. 3) and during spinal anaesthesia as in case 1 . Ischaemia decreased the activity. Intravenous edrophonium (Tensilon, $10 \mathrm{mg}$ ) produced no change in the clinical picture or the EMG.

Table 2 Nerve conduction studies-case 2

\begin{tabular}{|c|c|c|c|c|}
\hline \multirow[t]{2}{*}{ Nerve } & \multicolumn{2}{|c|}{$\begin{array}{l}\text { Distal latency } \\
(m s)\end{array}$} & \multicolumn{2}{|c|}{$\begin{array}{l}\text { Conduction } \\
\text { velocity } \\
\text { (metres/second) }\end{array}$} \\
\hline & Record & Normal & Record & Normal \\
\hline $\begin{array}{l}\text { Right median (motor) } \\
\text { Right median (sensory) } \\
\text { Right ulnar (motor)- } \\
\text { supraclavicular fossa } \\
\text { to above elbow } \\
\text { Right ulnar (motor)- } \\
\text { above elbow to } \\
\text { below elbow } \\
\text { Right ulnar (motor)- } \\
\text { below elbow to wrist } \\
\text { Right peroneal (motor) } \\
\text { Right sural (sensory) }\end{array}$ & $\begin{array}{l}5.5 \\
8.0 \\
\text { Unobtai }\end{array}$ & $\begin{array}{l}1.8-4.2 \\
3.4-6.8 \\
\text { inable }\end{array}$ & $\begin{array}{l}28 \\
38 \\
32\end{array}$ & $\begin{array}{l}45-75 \\
45-75 \\
38-65\end{array}$ \\
\hline
\end{tabular}

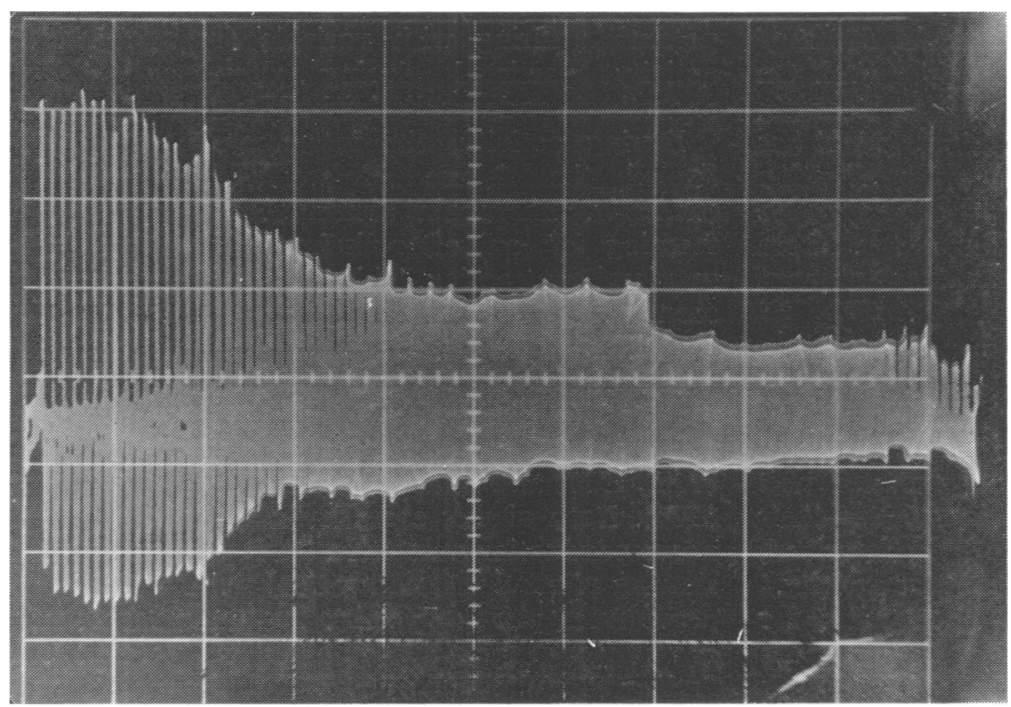

Fig. 2 Decrementing response observed in case 2 on stimulating the ulnar nerve at a rate of 50/second (amplitude: $100 \mu \mathrm{V} / \mathrm{cm}$; time scalc: $200 \mathrm{~ms} / \mathrm{cm}$ ). 


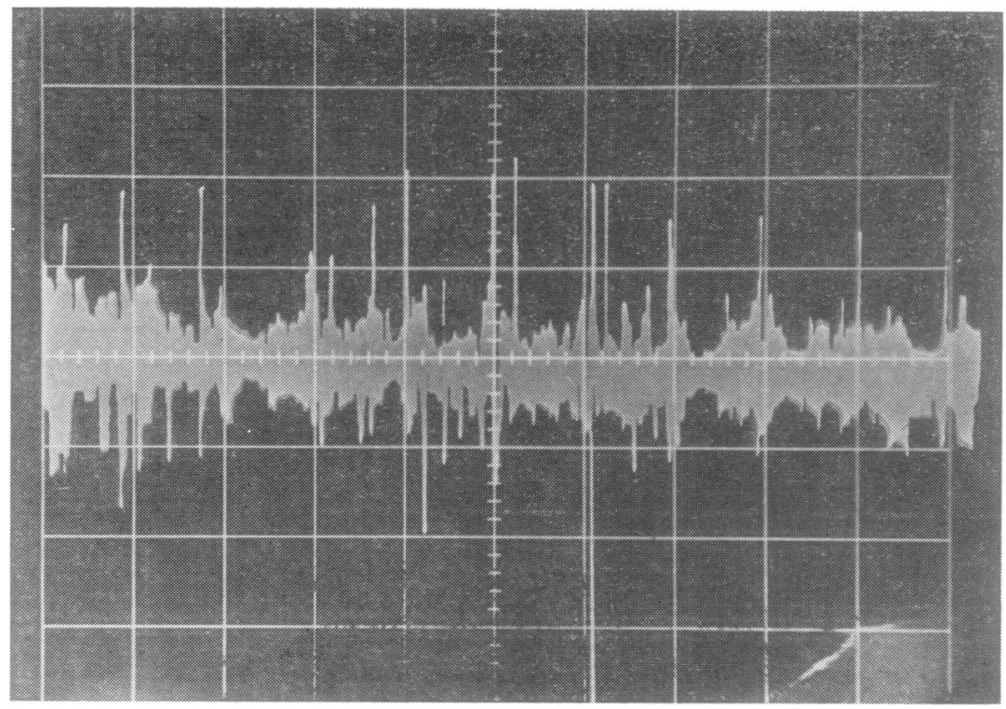

Fig. 3 Needle electromyagraphy at rest in case 2 revealing continuous electrical activity (amplitude: $100 \mu \mathrm{V} / \mathrm{cm}$; time scale: $200 \mathrm{~ms} / \mathrm{cm}$ ).

\section{Histology}

Biopsy of the peroneus brevis muscle and the musculocutaneous nerve of the leg, using the technique of Coërs and Woolf (1959), revealed no significant abnormalities on light and electron microscopy. Muscle histochemistry with ATPase, succinyl dehydrogenase, acid phosphatase, and cholinesterase stains were normal. Intravital methylene blue staining (Coërs and Woolf, 1959) demonstrated beading and collateral sprouting of terminal motor nerve fibres and multiple innervation of muscle fibres with varying morphology of the endplates (Fig. 4).

\section{Course}

The patient was treated with phenytoin, $500 \mathrm{mg} /$ day, reaching blood levels of 34 to $45 \mu \mathrm{g} / \mathrm{ml}$. On this regimen he has gradually regained strength, his reflexes have increased, and his myokymia has diminished. The slowing of the nerve conduction velocities and decrementing response to repetitive stimulation have not changed with therapy. There are no signs of phenytoin toxicity. Improvement has lasted for 24 months.

\section{Discussion}

Both patients fit the syndrome of "myokymia and impaired relaxation with continuous motor unit activity." They differ in two main respects-their weakness is more severe than has been described, and the weakness is relieved by repetitive activity.

In our cases the initial effect of exertion, for example, flexion of the elbow, was to aggravate the weakness. If the patient persisted, the strength of the action improved, slowly reaching normal or nearly normal power. That this is not part of a "warming up" process by which stiffness may be relieved in this and myotonic disorders (Brown, 1974) is suggested by its occurrence when weakness (case 2) or phenytoin (case 1) had abolished the stiffness. Transient fatigue with improvement after repetition has been described as an infrequent occurrence in myotonia congenita (Aminoff et al., 1977; Brown, 1974); however, the weakness of that disorder is maximal after rest whereas strength was improved by rest in both our cases.

The EMG data in our cases are similar to those in other reports. At rest there was continuous electrical activity, of full and fractionated unit potentials, firing with variable frequency. This activity persisted during sleep and spinal anaesthesia and was diminished by curare in case 1 .

During activity, the interference pattern reflected the degree of weakness appreciated clinically. The main abnormal feature was the forming of an after-discharge after a single maximal contraction. This after-discharge was not seen when strength had been restored by repetitive contractions.

Stimulation of the peripheral nerves has been reported to show slowing of motor conduction velocity (Wallis et al., 1970; Waerness, 1974; Irani et al., 1977) and prolonged motor latencies (Isaacs, 1961). Both of our cases had marked slowing of nerve conduction velocities. In one case we found slowing of conduction over the length of the ulnar nerve from plexus to wrist. The only evidence in any way suggestive of a junctional disorder is our finding of a decrementing response at stimulation 


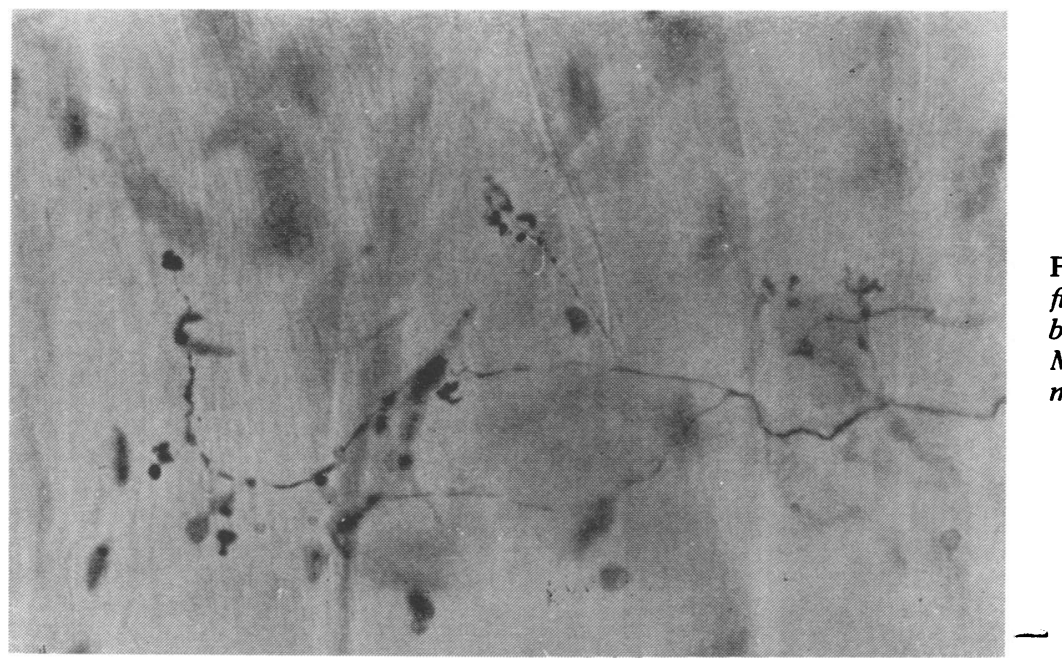

Fig. 4 Terminal motor nerve fibres in case 2 demonstrating beading and collateral sprouting. Methylene blue, original magnification $\times 400$.

rates of $50 / \mathrm{s}$ in one case. This may be due to fatigue of a damaged nerve (Simpson, 1962). We found no incrementing response as is seen in the myasthenic syndrome (Lambert and Rooke, 1965).

Nerve and muscle biopsies have shown normal muscle fibres in most cases. Lesions of the sural nerve have been reported in two cases. Segmental demyelination was reported by Welch et al. (1972) and degenerative changes in a few fibres were noted by Wallis et al. (1970).

In motor nerves the terminal fibres have been found abnormal by Isaacs and Frere (1974) and in our case 2. Mertons and Zschocke (1965) reported no abnormality of the motor nerve terminals in their one case. We were unable to find neuromuscular junctions in sections prepared for electron microscopy in our and other laboratories. Reduced numbers of vesicles and complex convoluted synaptic clefts on electron microscopy are described in one case (Sroka et al., 1975).

The balance of the evidence-the involuntary activity, the depressed reflexes, the proliferation and branching of the terminal motor nerve fibres, the abnormalities of conduction velocity-suggest that there is a lesion in the peripheral motor nerve.

The aetiology of this disorder is unknown. Most cases have no clear precipitating agent. Wallis et al. (1970) reported one case after exposure to the herbicide, dichlorophenoxyacetic acid (2, 4-D), and an animal model of this disorder produced by exposure to the drug 2-azaridinyl ethanol (2A-E). Neuromyotonia has been associated with occult neoplasm (Waerness, 1974). One case has been reported of myokymia localised to those muscles innervated by an injured nerve (Medina et al., 1976). Our case 2 had a history of treated syphilis and amphetamine abuse; however, there was no clear association between these problems and his neuromuscular disease which progressed despite his discontinuation of drug abuse..

Both phenytoin and carbamazepine have been shown to be effective therapy for this disorder (Isaacs, 1961; Wallis et al., 1970; Welch et al., 1972). Our cases improved remarkably with phenytoin but required high doses to achieve maximal improvement. They tolerated high phenytoin blood levels $(24-45 \mu \mathrm{g} / \mathrm{ml})$ without any signs of toxicity.

We acknowledge gratefully the collaboration of Dr T. Lentz and Dr G. Pappas, and we thank Dr G. Klingon for allowing us to study his patient. This work was supported in part by PHS Grant 5M01RR00047.

\section{References}

Aminoff, M. J., Layzer, R. B., Satya-Murti, S., and Faden, A. I. (1977). The declining electrical response of muscle to repetitive nerve stimulation. in myotonia. Neurology (Minneapolis), 27, 812816.

Brown, J. C. (1974). Muscle weakness after rest in myotonic disorders: an electrophysiological study. Journal of Neurology, Neurosurgery, and Psychiatry, 37, 1336-1342.

Coërs, C., and Woolf, A. L. (1959). The Innervation of Muscle: a Biopsy Study, pp. 1-11. Blackwell Scientific Publications: Oxford.

Irani, P. F., Purohit, A. V., and Wadia, N. J. (1977). The syndrome of continuous muscle fiber activity. Acta Neurologica Scandinavica, 55, 273-288.

Isaacs, H. (1961). A syndrome of continuous muscle- 
fibre activity. Journal of Neurology, Neurosurgery, and Psychiatry, 24, 319-325.

Isaacs, H., and Frere, G. (1974). Syndrome of continuous muscle fiber activity. South African Medical Journal, 10, 1601-1607.

Lambert, E. H., and Rooke, E. D. (1965). Myasthenic state and lung cancer. In The Remote Effects of Cancer on the Nervous System, pp. 67-80. Edited by W. R. Brain and F. Norris. Grune and Stratton: New York.

Medina, J. L., Chokroverty, S., and Reyes, M. (1976). Localised myokymia caused by peripheral nerve injury. Archives of Neurology (Chicago), 33, 587588.

Mertons, H. G., and Zschocke, S. (1965). Neuromyotonia. Klinische Wochenschrift, 43, 917-925.

Simpson, J. A. (1962). The clinical physiology of the lower motor neuron. Developmental Medicine and Child Neurology, 4, 55-64.

Sroka, H., Bornstein, M., and Sandbank, U. (1975). Ultrastructure of the syndrome of continuous muscle fibre activity. Acta Neuropathologica, 31, 85-90.

Waerness, E. (1974). Neuromyotonia and bronchial carcinoma. Electromyography and Clinical Neurophysiology, 14, 527-535.

Wallis, W. E., Van Poznak, A., and Plum, F. (1970), Generalized muscular stiffness, fasciculations and myokymia of peripheral nerve origin. Archives of Neurology (Chicago), 22, 430-439.

Welch, L. K., Appenzeller, O., and Bicknell, J. M. (1972). Peripheral neuropathy and myokymia, sustained muscular contraction, and continuous motor unit activity. Neurology (Minneapolis), 22, 161-169. 